
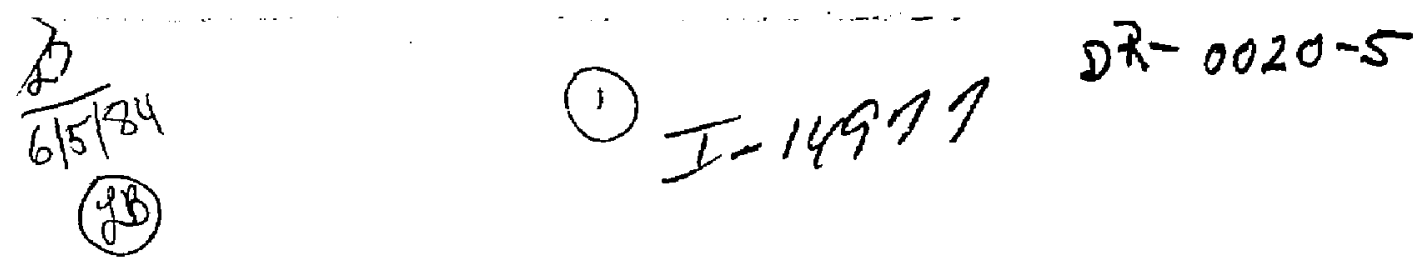

UCID- -20026

DES4 012413

\title{
IMPURITY CHARACTERISTICS OF TMX-U
}

S. L. Allen

T. L. Yu

T. J. Nash

May 1, 1984

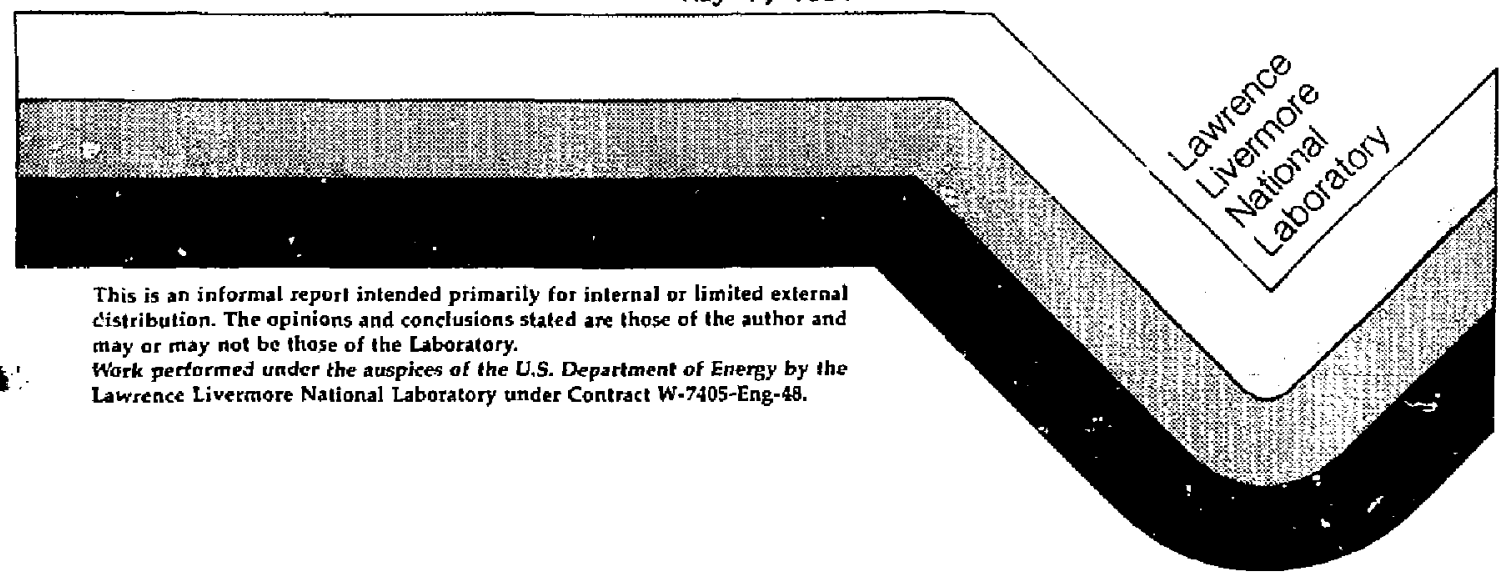

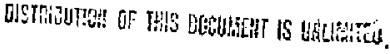




\title{
ABSTRACT
}

A study of the concentrations, radiated power, and sources of impurities in the TM $\mathrm{X}-U$ plasma is presented. Three extreme ultraviolet (EUV) instruments were used: one monachromator that measures time-resolved radial profiles of a particular impurity line, and two time-resolving spectragraphs that measure the time histories of several impurity lines in a single shot. The major impurities found in the TMX-U plasila are carbon, nitrogen, and oxygen. Titanium and silicon are also observed. The impurity concentrations are low, less than $1 \%$ and the radiated power is a small fraction of the input power. The major source of axygen and nitrogen is the neutral beam injector. Walt conditioning, such as glow discharge cleaning and titanium gettering, decreases the impurity concentrations, particularly carbon. There does not seem to be an accumulation of impurities in the thermal barrier region under the present plasma conditions. Future experiments are discussed.

\section{DISCLAIMER}

\begin{abstract}
This report was prepured as an aceount of work sponsored by an agency of the United States Government. Neither the United States Government nor kny agency thereof, nor any of their employees, makes any warranty, express or iroplied, or assumes any kgal liability or responsibility for the accuracy, completeness, or usefulness of any information, apparatus, product, or process disclosed, or represents that is use would not infringe privately owned rights. Reference hercin to any specific commercial product, process, or service by trade name, trademark, manufacturer, or atherwise does not necessarily constilute of ittiply its endorsement, recommendation, or favoring by the United States Government or any ageacy therect. The views and opinions of authors expressed berein do not nesessarily state or rellect those of the United Strtes Government or any agency thereor.
\end{abstract}

*The Johns Hopkins University, Baltimore, Maryl and.

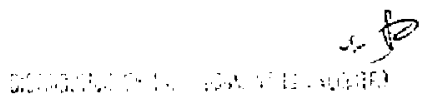




\section{INTRODUCTION}

In a large fusion device, impurities can be introduced into the plasma from the walls, limiters, and other sources such as neutral beams. These impurities can cause significant power loss by radiation. ${ }^{1-5}$ In addition, impurities can influence the plasma potential and its stability. For these reasons, many experiments have been carried out on both "naturally occurring" and artificially injected impurities. In the past few years, in particular, the amount of impurity research on mirror devices increased substantialiy, with a concomitant increase in sophistication of diagnostics instrumentation.

In this report, we describe impurity behavior and discuss specific experiments on impurities in the Tandem Mirror Experiment-Upgrade (TMX-U) ${ }^{6}$ at the Lawrence Livermore National Laboratory (LLNL). The TMX-U is the third such device at LLNL; previous experinients were carried out on the $2 X I I B^{7}$ (single) mirror machine and on TMX.8,9 The TMX was a tandem mirror composed of two end plugs--each similar to 2XIIB--connected by a central celi. The end plug density was greater than that in the central cell, resulting in a potential difference that enhanced the axial confinement. Because these previous results are relevant to TMX-U, it is useful to briefly describe them first.

On 2XIIB, we used an extreme ultraviolet (EUV) monochromator to determine that the neutral beam injectors were the major source of oxygen in the plasma. ${ }^{10}$ The impurity concentrations were low in this device, 11 and the radiated power was a small fraction of the total input power (Ref. 12 presents even more detail about the $2 X I I B$ results). Furthermore, the low-energy impurity ions were shown to have a short confinement time, presumably because of the rapid scattering times of impurities in a magnetic mirror. Carbon, nitrogen, and titanium were also identified as impurities but their concentrations were less than oxygen concentrations.

The next set of experiments were carried out on TMX. A single (radial) chord EUV monochromator and two spatially imaging EUV monochromators were used to obtain time-resolved impurity radial profiles of both the central- and end-cell plasmas. Again, the major impurity found was oxygen, and the impurity characteristics of the TMX plug were similar to the 2XIIB results. ${ }^{13}$ The central-cell oxygen concentration was approximately $0.3 \%$, and lesser 
amounts of nitrogen, carbon, and titanium were observed. ${ }^{14}$ One source of titanium was from the stream guns used to initiate the target plasma in the end plugs. The total radiated power from impurities in the central cell was less than 20 to $30 \mathrm{~kW}$. Impurity injection with gas puffing indicated that impurities do not accumulate in the central cell of a tandem mirror. Furthermore, the radial transport of impurities was outward. The edge plasma seemed to shield the plasma from penetration of impurities to the plasma core. ${ }^{15}$ Gettering lowered the impurity levels in the central cell.

The current experiment at LLNL is TMX-U; the object. of this experiment is to confine the plasma by means of a thermal barrier. 16 The thermal barrier is formed with a combination of electron-cyclotron resonant heating (ECRH), ion-cyclotron resonant heating (ICRH), and neutral beam injection. This allows the central-cell plasma to be more dense than the end plugs, yet still have axial confinement. Two EUV time-resolving spectrographs and a spatial-imaging monochromator measured the impurity emissions in the end plugs and central cell of this experiment.

Briefly, the initial impurity results from TMX-U are similar to the TMX results. Namely, the central-cell impurity concentrations continue to be low; less than $1 \%$ of carbon, nitrogen, and uxygen. Smaller amounts of titanium and, in some cases silicon, were observed. The end-plug impurity measurements indicate that oxygen (and perhaps nitrogen) is injected with the neutral beams. The infurity concentrations are influenced by wall conditioning, especialiy glow-discharge cleaning and titanium gettering. Under the present plasma conditions, impurities da not seem to accumulate in the thermal barrier region. This is consistent with a mode ${ }^{17}$ that predicts a small accumulation with the current barrier depth $(500 \mathrm{eV})$ and the average impurity charge state $(Z=4)$ observed in TMK-L.

The instruments used to make the measurements are described in Section II. The impurity densities and radiated power measurements are detiiled in Section III. The sources of impurities and the influence of wall conditions on impurity characteristics are also discussed in section iII. In the final section we summarize the results, discuss briefly the measurements during thermal barrier operations, and describe ongoing and future experiments. 


\section{EXPER IMENTAL DETAILS}

The goals of the impurity studies on TMX-U include the following:

- Identification of the important impurity species.

- Measurement of the densities of the impurities as a function of time and space.

- Estimation of the radiated power from these impurity species.

- Understanding the sources of the impurities and the possibilities for control, particularly in the thermal barrier region.

The spectroscopic instrumentation was chosen with these goals in mind. That is, both spatial and spectrat resoiution are required. For this reason, a spatially imaging monochromator (the same one that was used on TMX) and two multi-element spectrographs were employed. The first instrument measures a time-resolved radial profile of one impurity line. The other two instruments measure time-resolved spectra along the chord.

The design and construction of the spatially imagina monochromator, shawn in Fig. 1, is discussed in detail elsewhere. ${ }^{18,19}$ Briefly, the instrument is a $0.4-m$ normai-incidence monochromator with a multi-element detector. Spatial imaging is accomplished by using the entrance slit as a one-dimensional analog of a pinhole camera; each position in the plasma has a corresponding point on the detector. The desired wavelength is selected by rotating the grating, which is remotely controlied by a precision grating drive. The monochromatic image of the plasma is thereby directed on the detector. The detector consists of a pair of microchannel plates in the chevron configuration; 22 discrete anodes are placed behind the plates to collect the charge. The charge from each anode is measured by a pulse amplifier and discriminator circuit and a counter. The digital data is then stored in a local CAMAC memory. This memory can be read either by a local microcomfuter or by the main $T M X-U$ database computer. This instrument is obsolutely calibrated, both in wavelength (the grating position) and intensity the latter by comparison with intensity standards of the National Bureau of standards (NBS)].

The two time-resolving spectrographs perform similar functions, but each systsm is outimized for a particular wavelength region: the Normal Incidence 


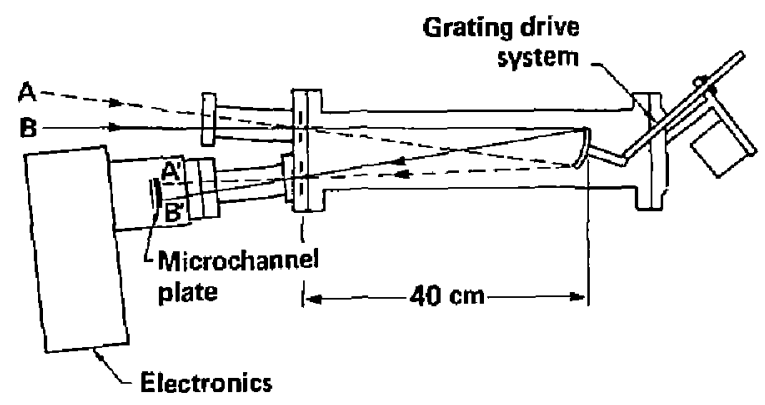

Figure 1. The 0.4-m monochromator with 22-channel Spatial Imaging Detector System (SIDS). The two rays $A$ and $B$ from the plasma are imaged at the detector to the two points $A^{\prime}$ and $B^{\prime}$, respectively. The instrument has its ow' separate high-yacuum system and is absolutely calibrated in wavelength and intensity. 
Spectrograph (NIS) for 300 to $1600 \mathrm{~A}$, and the Grazing Incidence Spectrograph (GIS) for 20 to $300 \AA$. The normal incidence system is the result of a cooperative experiment with The Johns Hopkins University (for details, see Ref. 20). An outline drawing of the apparatus is shown in Fig. 2, and a schematic of the optical system is given in Fig. 3. Note that by tilting the spectrograph, one of seven different gratings can be selected. In this manner, different wavelength regions can be studied with a particular spectral resolution. Table 1 indicates the wavelength coverage and the corresponding spectral resolution for each grating. The detector system consists of an image intensifier-converter and a 1024-channel photodiode array. An electronic controller reads out this array and the data is stored in a local CAMAC memory. The data is then read either by a local microcomputer or the TMX-U data acquisition system. In addition, the detector array can be programed in a line-select mode so that a fixed number of lines (channels in the detector) are read, and the rest of the data is discarded. This allows the time resolution of the system to increase from $4 \mathrm{~ms}$ for the whole array to about $1 \mathrm{~ms}$ for the line-select mode. The instrument can be scanned radialiy by remote control so that shot-by-shot radial data can be obtained. The spectrograph has been absolutely calibrated at the SURF facility of NBS and also by comparison with NBS reference photodiodes at The Johns Hopkins University.

The other spectrograph system is the result of a cooperative experiment with E-Division at LLNL. A schematic of the GIS is shown in Fig. 4. The design is similar to the NIS, except only a single grating is used, and the EUV emissions strike the grating at grazing incidence. This is necessary to obtain a useable grating efficiency in the $20 \AA$ wavelength region. As shown in the figure, a separate calibrated $x-r a y$ source can be mounted an the spectrograph for absolute calibration. The detector system is controlled by a LSI-11 computer having local data acquisition and storage. The time resolution of this sytem is about $4 \mathrm{~ms}$. The focal length of the spectrograph is $2.2 \mathrm{~m}$. Because the support structure is correspondingly large, it is not practical to perform radial scans of the TMX-U plasria. More details are presented in Ref. 21 . 


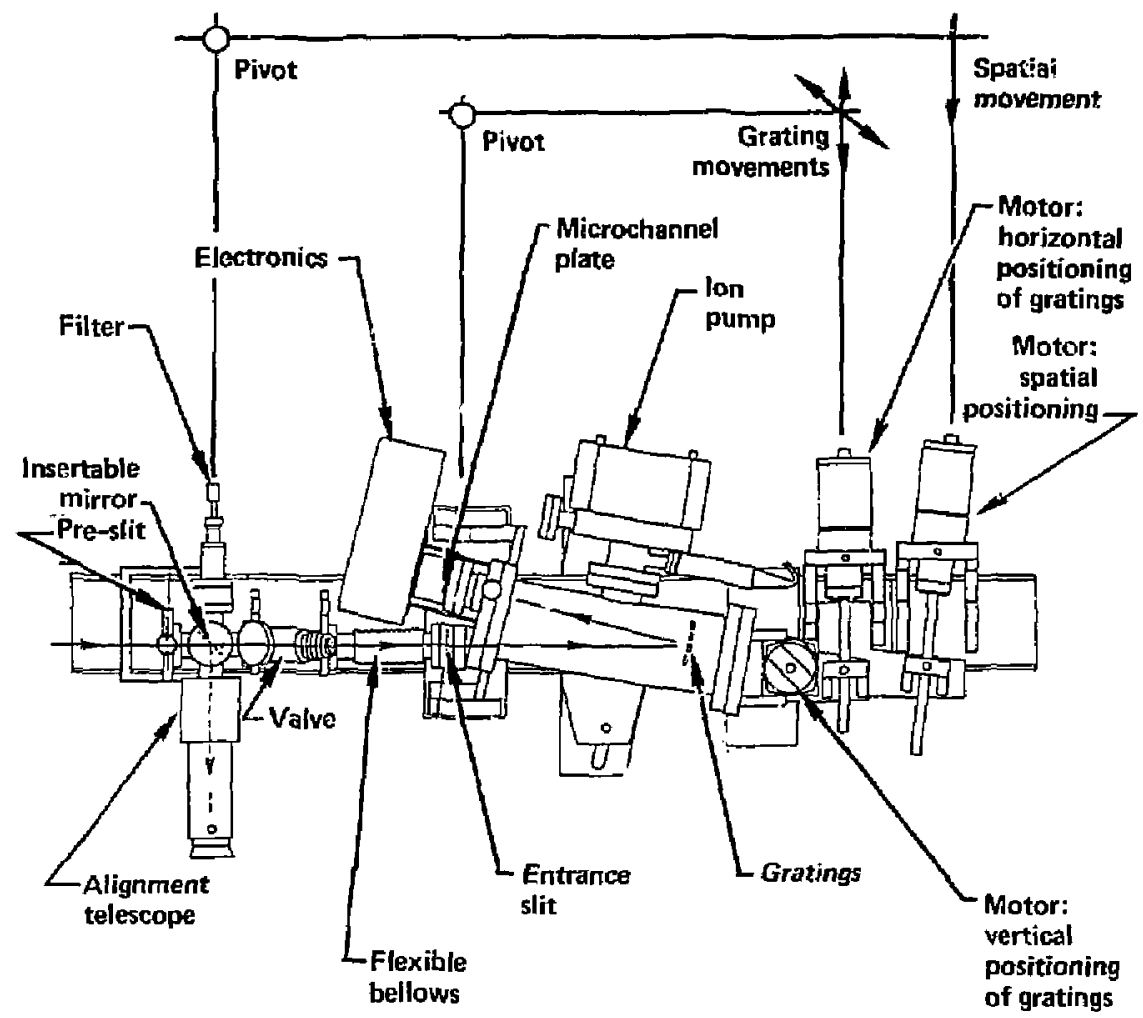

$\longmapsto \quad 40 \mathrm{~cm} \quad \longrightarrow$

Figure 2. The 0.4-m Extreme Ultraviolet (EUV) Spectrograph and 1024-channe? detector system. Note the two pivot points and two positioning systems; one selects the grating that views the plasma, the other tilts the whole instrument so that radial scans can be performed. A separate ajignment telescope positions the instrument on TMY-U. 


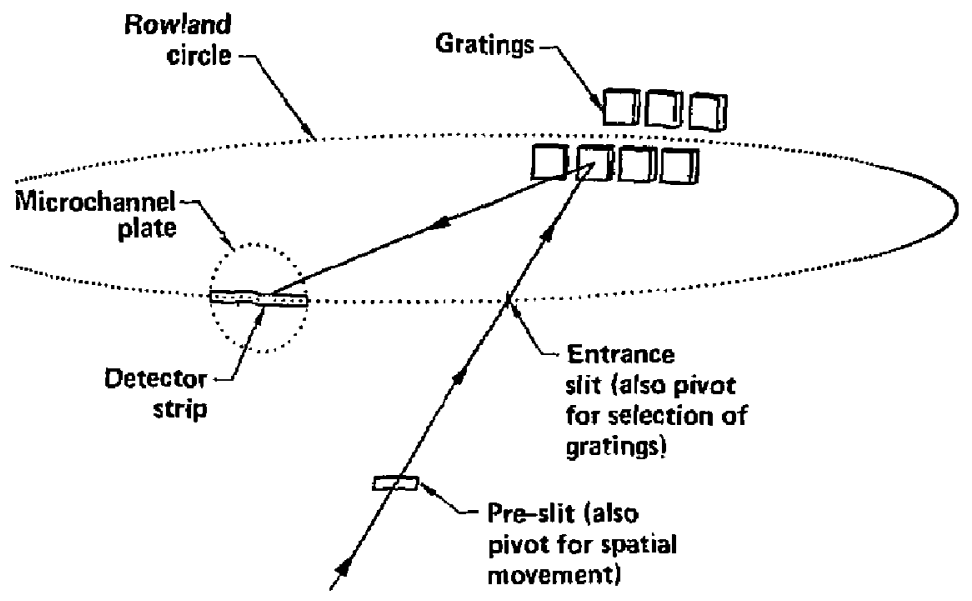

Fiqure 3. Optical system of the Normal Incidence Spectrograph (NIS). One of the seven diffraction gratings is selected by tilting the instrument about the entrance slit. The entrance slit, gratings, and detector system are positioned on the Rowland circle to insure that the spectra are focused. 
Table 1. Instrument parameters for Norma Incidence Spectrograph (NIS), Grazing Incidence Spectrograph (GIS), and Spatial Imaging Detector System (SIDS).

\begin{tabular}{|c|c|c|c|c|}
\hline Parameter & \multicolumn{2}{|l|}{ NIS } & GIS & $\begin{array}{c}\text { 22-Channe1 } \\
\text { SIDS }\end{array}$ \\
\hline Spectral rarge in $A$ & $210-1845$ & 4.1 & $20-300 \quad 0.5$ & $300-1700 \quad 0.2$ \\
\hline (resolution in $A$ ) & $1370-2200$ & 2.7 & $300-2000 \quad 2.0$ & ( 1.0 normally used) \\
\hline & 290 to 560 & 0.7 & & \\
\hline & 550 to 830 & 0.7 & & \\
\hline & 810 to 10800 & 0.7 & & \\
\hline & 1035 to 14601 & 1.0 & & \\
\hline & 1450 to 18701 & 1.0 & & \\
\hline Time resolution & $1-4 \mathrm{~ms}$ & & $4-5 \mathrm{~ms}$ & $125 \mu \mathrm{S}$ (Typicalty $/ \mathrm{ms}$ ) \\
\hline Spatial scanning & Shot-by-shot & & $\cdots$ & Single shot \\
\hline Calibration & NBS absolute & & Proportiorial & NBS absolute \\
\hline & calibration & & counter & calibration \\
\hline Detector & Image converter & & Image converter & Microchannel \\
\hline & (1024-channe) & & (1024-channe) & plate \\
\hline & Reticon) & & Reticon) & \\
\hline Location & Central cell & & Central cell & Central cel? \\
\hline & or plug & & & or plug \\
\hline
\end{tabular}




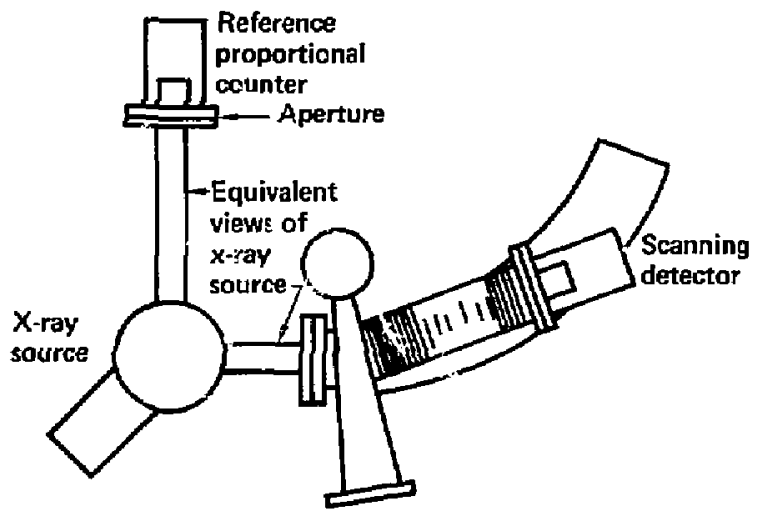

Figure 4. Grazing Inciderce Spectrograph (GIS) and its 1024-channel detector and the $x-r a y c a l i b r a t i o n$ source. A reference proportional counter can also mileasure the $x$-ray source for absolute calibrai iun. When jnstalled on TMX-U, tht: $x-r$ ay source is removed. The plasfa $l$ ight strikes the grating at grazing incidence to optimize the efficiency of the system in the 20 \& syectrai region. 
The locations of the initruments are shown in Fig. 5. Because the GIS is sa large, it views the central-cell plasma from the north side of the machine along a horizontal chord. The NIS and the spatial imaging detector system (SIOS) can be mounted so they view either the end-cell) or the ientral-cell piasma. With TMX-U at vacuum, these locatiors can be switched during an experimental cycle. The end-plus locations have sufficient optical access so that either radial or axial scaris of the thermal barrier region can be obtained. The central-celt location also allows access so that radial emission profiles can be measured.

\section{EXPERIMENTAL RESULTS}

Calculations of Impurity Concentrations and Radiated Power

The spectra obtained from the EUV instrumerts are used to calculate the concentration and radiated power of each impurity species. Because of the wide spectral range of the normal incicence spectrograph, it was used as the major source of data. The other instruments were l'sed to slibstantiate assumptions necessary to interpret the spectra and to check the results. A typical snectra obtained in the centrat cell during slashing-ion operation 22 is shown in Fig. 6; this covers the spectral region from $400101600 \mathrm{~A}$. Emissions from carbon, njtrogen, and oxygen can be identified.

The details of the calculations performed are presented in Appendix A. In summary, the cunceritration and radiated power from each iunization state of each inpurity species (e.g., C IV, or three times ionized carbon) is calculated from the line spectra, with the electron density and temperature as inpits. Some assumptions are made on the radial distribution of the impurities. In most cases, these assumptions do not affect the results greatiy, as similar results are abtained with other reasonable motels. The contributions from each ionization state are then sumfed together to obtain the total concentration and radiated power of one impurity species, such as carbon. These results are shown in Jable 2. Note that the radiated power and the concentration is smalt in each case. 


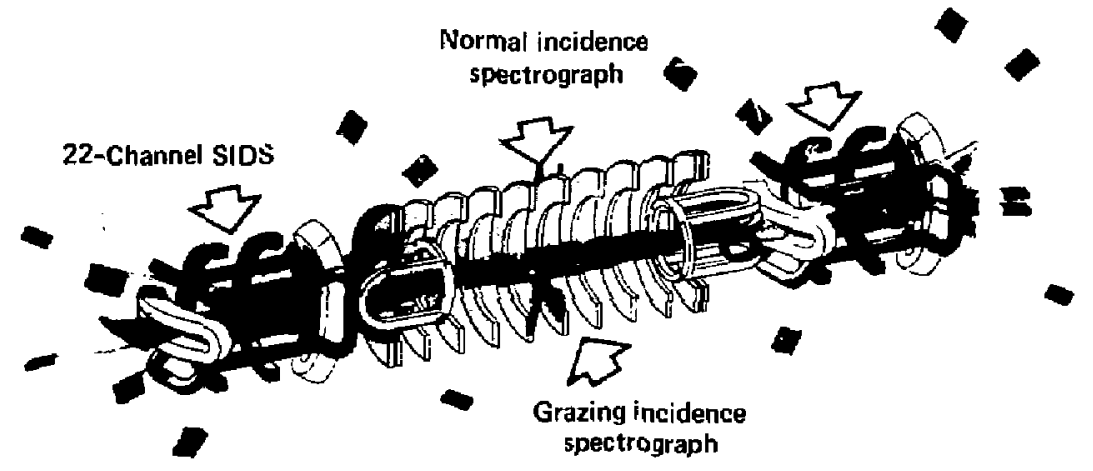

Fiqure 5. The locations of the spectroscopic instruments on TMX-U. Because of its size, the Grazing Incidence Spectroaraph is located on the side of the central celi and views the plasma along a horizontal chord. The other two instruments can be located either in the end cell or in the central cell (at the locations of the arrows on the top of the machine). Their positions can be changed while TMX-U is under vacuum. 


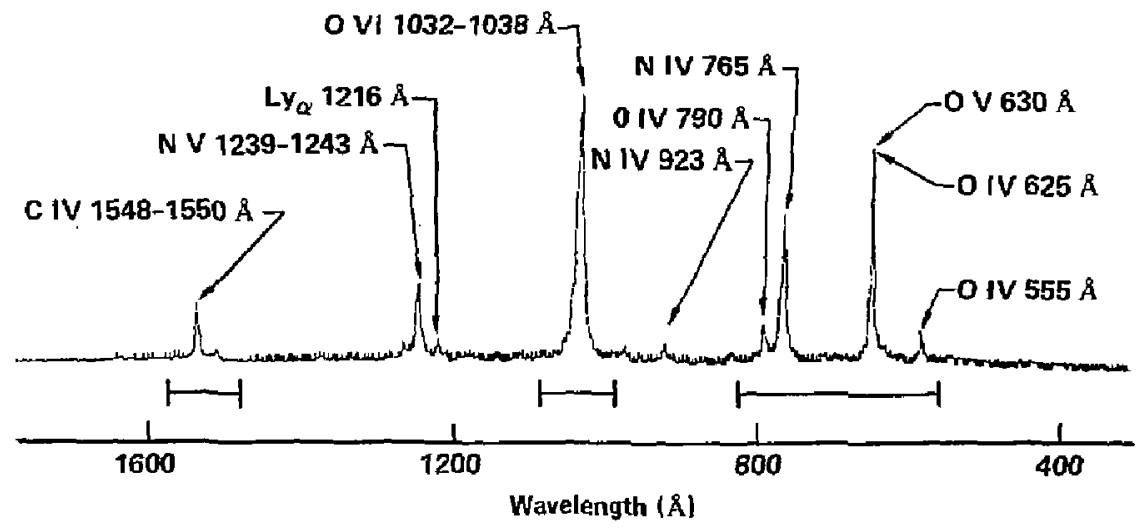

Figure 6. Typical extreme uTtraviolet spectrum of TMX-U central-celi plasma taken with the Normal Incidence Spectrograph during sloshing-ion operation. A time point near the steady-state portion of the plasma shot was selected. The solid bars illustrate how separate lines can be selected and measured with higher time resolution. This spectral range is used to determine the impurity concentrations and radiated power. 
Table 2. Impurities calculated ${ }^{a}$ for the TMX-U central cell during typical sloshing-ion operations. 23

\begin{tabular}{lcc}
\hline Impurity & Concentration (y) & Radiated Power (kW) \\
\hline Carbon & 0.06 & 0.3 \\
Nitrogen & 0.17 & 0.7 \\
Oxygen & 0.82 & 2.7 \\
\hline
\end{tabular}

\footnotetext{
amounts include only the inpurity states measured by the NIS, that is, the ionization states up to C IV, N $V$, and 0 VI. Emissions from the next higher ionization states-C V, N VI, and 0 VII--are outside the wavelength region of this instrument but can be measured by the GIS.
}

Note that the amounts in Table 2 include only the measured impurity states from the NIS spectra, that is, the ionization states up to $C$ IV, NV. and 0 VI. Emissions from the next higher ionization states-- $C V, N V I$, and 0 VII--are outside the wavelength region of this instrument but can be measured by the GIS. So far, no emission lines from these ionization states have been observed, but they are expected to be very weak at the present electron temperature. This means that some of the impurity density may be in these states, but it can oniy be approximated because of the weak emission from these states. A rough estimate based on the ratios of the measured ionization states is given in Table 3 . The uncertainty in these estimates is at least a factor of two. It is impartant to note that these states add to the impurity concentration, but they do not add an appreciable amount of radiated power, because of the very weak emissions. Nevertheless, even with the addition of these states, the concentrations are small, less than $1 \%$.

The spectra obtained from the GIS was used to determine the radiated power from titanium emissions in the central cell. (A calculation of the density is difficult, as the excitation rates are not well known for some of 
Table 3. Total impurity concentrations including an estimate of the concentrations of $C V, N$ VI, and 0 VI based on ratios of the measured ionization states.

\begin{tabular}{lc}
\hline Species & Concentration $(\%)$ \\
\hline Carbon & 0.9 \\
Nitrogen & 0.5 \\
Oxygen & 0.9 \\
\hline
\end{tabular}

the emission lines.) A sample spectra showing the emissions near $100 \mathrm{~A}$ is presented in Fig. 7; emissions from $\mathrm{Ti} V$, and $\mathrm{Ti} V I$, and $\mathrm{Ti} V I I$ have been identified. The calculated brightness of these lines is small, so that the total estimated radiated power from titanium emissions is only about $1 \mathrm{kw}$. The sources of titanium impurities are discussed further in the next section.

A simpler calculation was performed for the purpose of comparing the end-cell impurity levels on TMX-U with those of TMX. The oxygen brightnesses were scaled, taking the electron density and plasma voiume into account on each machine. ${ }^{24}$ Within a factor of two, the results compare iavorably, indicating that the fraction of the beam current that is oxygen is simiiar in the two cases.

\section{Sources and Control of Impurities}

As mentioned above, the neutral beams are the major source of oxygen in the plasma. As measured on $2 X I I B$ and $T M X$, this oxygen is an anergetic component of the neutral beam and is usually about $1 \%$ of the injected beam current. The data of Fig. 8 suggest that nitrogen may alco be associated with the neutral beams; the time histories of carbon, nitrogen, and oxygen ionization states are presented. Note that when the centrai-cell neutral beam are turned off, the nitrogen emission decreases along with the oxygen, while the carbon emission remains constant until the plasma is turned off. This 


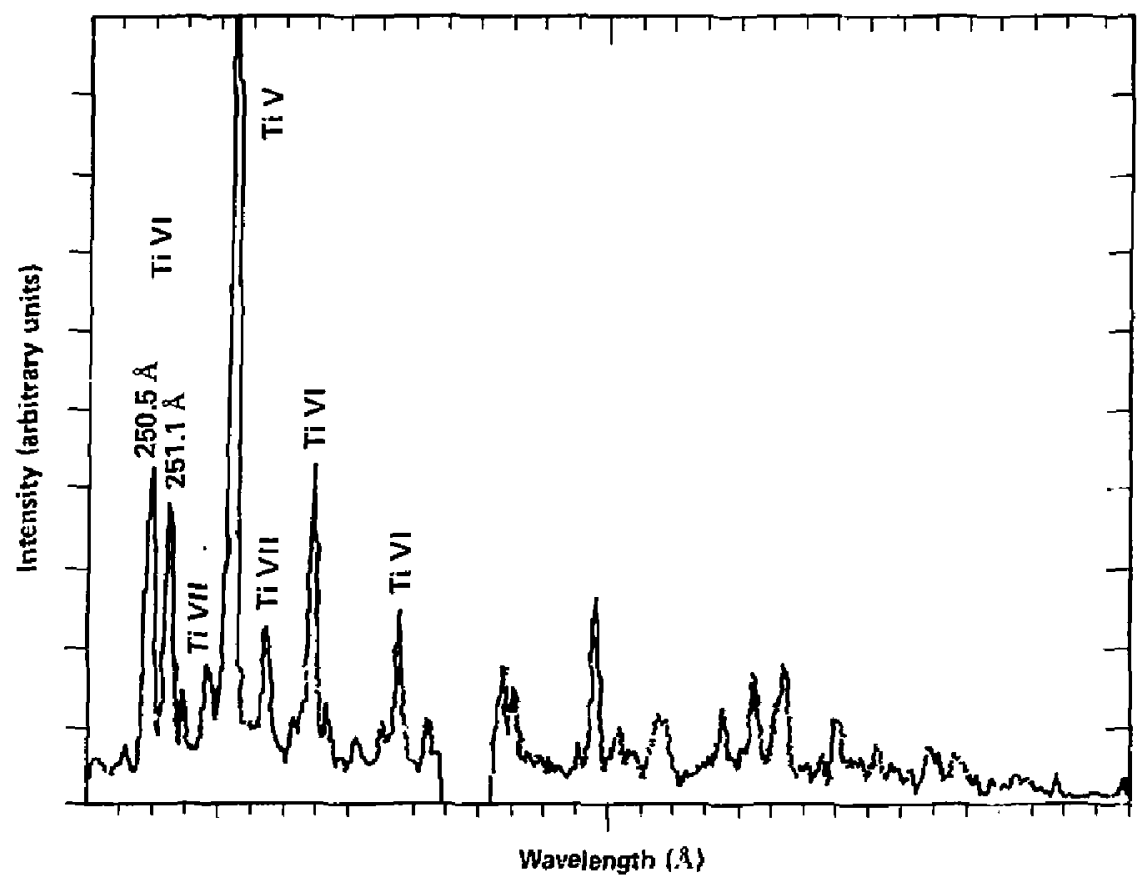

Figure 7. Titanium emissions spectra obtained from the Grazing Incidence Spectrograph. A time point near the steady-state portion of the plasma was selected. Note emissions from titanium ionization states. The brightness of these emissions is small so the radiated power is usually less than $1 \mathrm{~kW}$. 


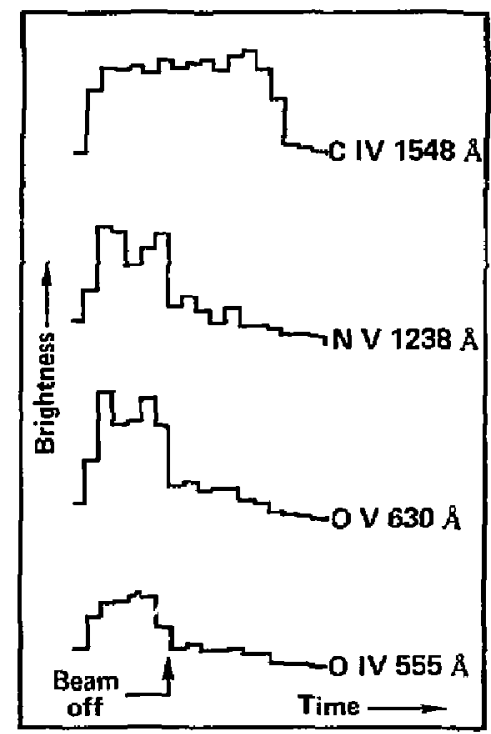

Figure 8. Brightness for four impurity ionization states as a function of time as measured by the Normal Incidence Spectrograph in the central cell. The neutral beams were turned off at the time indicated by the arrow. The oxygen and nitrogen ionization states decrease at this time, while the carbon remains constant. This indicatas the neutral beams are a major source of nitrogen and oxygen in the plasma. 
suggests that at least part of the nitrogen source is due to the neutral beams. The spectral resolution of the normal incidence instrument is not sufficient to determine if these emissions are Doppler broadened and thus an energetic component of the beam, or are a cold streaming gas from the source,

The control of oxygen impurities in the beams was accomplished by titanium gettering of the arc chambers. Tests show that the oxygen fraction can be reduced at least an order of magnitude ${ }^{25}$ by this technique, anc the sources will run reliably after some adjustment. Titanium getters are installed on all the TMX-U neutrál-beam sources but have not tien used to date because the impurity emissiors are quite low.

The major source of carbon impurities seems to be the walls of iny-L. As shown in Fig. 9, the vacuum vessel is made up of two liquid-nitrogei-cooled liners, and a plasma warm wall. All of these surfaces are covered with titanium gettering before each piasma shot. Methane is only loosely bound to the cold liners, as shown by the time history of the pressure in Fig. 10. The data were obtained during a $T M X-U$ experiment run; note that methane is liberated shortly after the liners begin warming. The methane in the residual gas and other hydrocarbons bound to the walls is assumed to make up the carbon source.

For this reason, glow discharge cleaning was used to reduce the surface carbon concentration. Shown in Fig. 11 is the 16-amu signal from a residual gas analyzer during glow discharge cleaning; this signal is composed mostly of methane. Methane and other hydrocarbons are liberated when the glow discharge is turned on, indicating that carbon is being removed from the surfaces. The decrease in the rate with time is similar to the results obtained in tokamak cleaning runs.

The decrease in the surface carbon concentration influences the plasma radiated power and the impurity concentrations. We performed a specific set of experiments to determine if glow discharge cleaning could be used instead of gettering during sloshing-ion operation of TMX-U; these experiments are discussed in Refs. 26 to 28 . Brjefly, glow discharge cleaning of the plasma wall could be used to obtain neutral-beam-sustained sloshing-ion plasmas. That is, the plasma would sustain with the neutral beams after the ECRH plasma target was turned off. Carbon emissions decreased after glo: discharge 


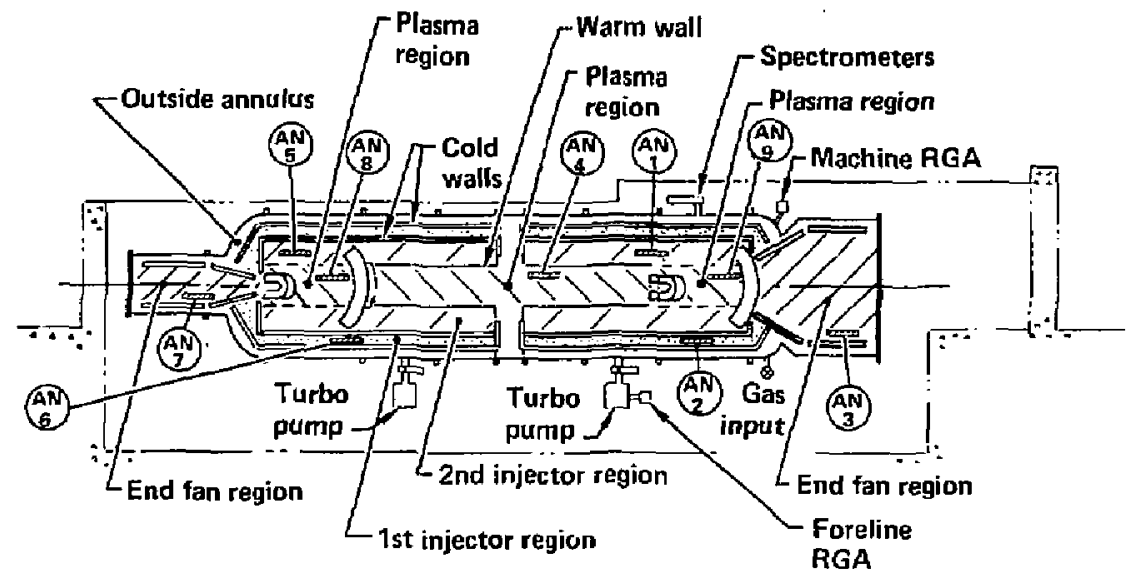

Figure 9. The TMX-U vacuum vessel and glow-discharge cleaning system. Hote the two liquid-nitrogen-cooled liners and the inner-plasma warm wall. Titanium is deposited on these walls before each plasma shot. The flow discharge cleaning anode locations are labeled AN\#1 to AN\#9. 


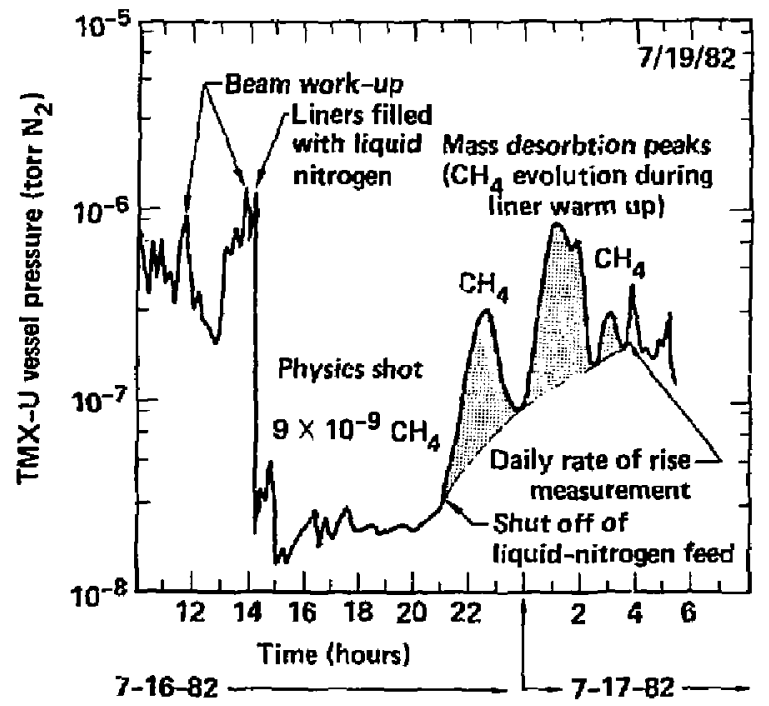

Figure 10. Pressure-time profile during a TMX-U run. The pressure increases when the liquid nitrogen that is used to fill the liners is shut off. Residual gas analysis shows that a large fraction of this rise is due to methane coming off the liners. In several cases, methane is the major background gas component. These findings indicate that the walls are a source of the observed carbon impurities in the plasma. 


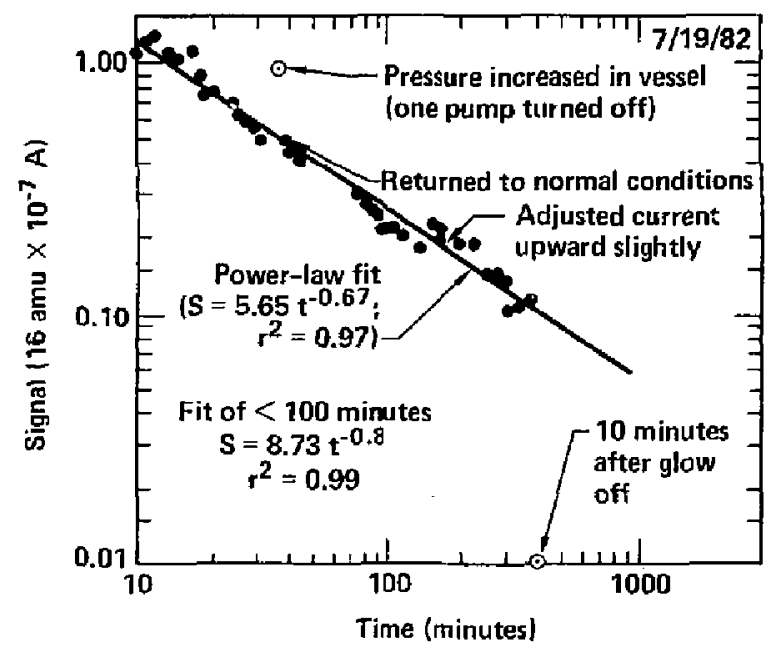

Figure 11. The 16-amu signal as a function of time during a glow-discharge cleaning run. This signal is mainly due to methane, but ather hydrocarbons are also liberated during glow-discharge cleaning. These resuits indicate that carbon is being removed from the walls. 
cleaning. Carbon emissions also were observed to fecrease with time during the run, suggesting that the plasma was also doing wall conditioning. This also is consistent with tukamak experience. We plan to test this result under thermal barrier canditions.

As expected, gettering also decreases the impurity concentrations in the plasma. At the end of the experiment discussed above, the plasta wall was gettered. Carbon IV emissions decreased by about a sactor of 5 at this time. Furthermore, this decrease persisted for several shots even after the gettering was stopped. This is also consistent with experiments carried out on tokamaks.

Final7y, it is important to discuss the sources and betavior of cther impurity species in the plasma. Small silicon emissions were identified duril? some experimental runs. In one instance, we observed a large amount of silicon when an ECRH window was damaged. Because the emissions are usually small ar:d appear only occasionally, silicon impurities are not considered to be a problem. Titanium emissions also were identified. Previous experiments on TMX show the titanium washer staci: guns can be a source of titanium. Shown in Fig. 12 are the radial profiles of titanium emission in the TMX end cell as a function of time. In TMX-U, ECRH is used almost exclusively to create a target plasma. This eliminates one source of titanium. The other obvious sulurce is the titanium gettering on the walls. Evidently, the plasma is sufficiently separated from the wall so that under the present conditions this is not a major source.

\section{CIISCUSSION OF RESULTS}

A.s discussed above, the spectroscopic measurements of 5 loshing-ion plasmas show that the impurity concentrations are 10\%, less than about $1 \%$, and the radiated power is small. oxygen and nitrogen are introduced by the neutra 7 beams, and one source of carbon is the TMX-U wall. Wall conditioning is important in the control of impurities. Titanium and silicon were abserved under some situations.

In addition, impurity measurements were made in the TMX-U end plug during thermal barrier cperation. No large increases in the impurity emissions were 


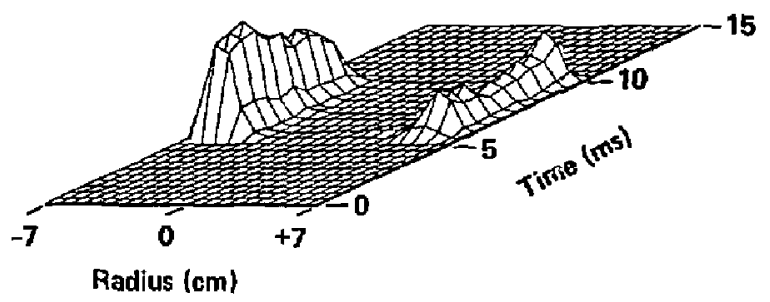

Figure 12. Radial brightness profiles for titanium emission as a function of time. These data were obtained on TMX, where stream guns were used to

generate a plasma target. On TMX-U, ECRH is used to start up the plasma, thus eliminating one source of titanium. 
observed when the axial plasma losses were reduced" ${ }^{29}$ (so-cailed "plugging"). When gradual increases were observed, it was felt that the electron temperature or density was increasing at the :ame time, thus influencing the impurity radiation. One model of impurity trapping in a thermal barrier is presented in Ref. 77 . The resuits of these calculations indicate that under che present plasma conditions, no large accumulation wouls be expected. To ba sure, spectroscopic messurements will continue in the end piug to check for impurity accumulation as the plasma parameters (i.e., the depth of the thermal barrier) increase.

The impurity measurements also were used to make qualitative estimates of plasma parameters, in particular, the electron temperature. The normalized time histories of four ionization states in the TMX-U end plug are shown in Fig. 13. These data were obtained with the spatial imaging monuchromator during a jet of four similar plasn.a shots. Under these low por r ECRH conditions ( $<50 \mathrm{~kW})$, the higher ionization states appeared after the lower ones; note this is independent of impurity species. Because the ionization rate increases with increasing temperature, this measuremeri implies that the electron temperature is increasing in time. Although the measurement is qualitative, it is an important diagnostir tool.

Similar methods were used in the ientral cell; titaniun and oxygen ionization states ${ }^{7}$ were measured by the time-resolving spectrographs. Several ionization states can be observed at one time, so relative changes in the electron temperature can be distinguished from increases in impurity concentration. That is, we can determine if the ratio of the ionization states is increasing (implying a change in temperature) or if all of the ionization states are increasing uniformly while the ratio remains constant (implying a source of impurities). As long as the confinement time of all the states is similar and constant in time, a qualitative estimate of the increase or decrease in the electron temper ature can be obtained. This has been particularly important during central-cell heating experiments.

Finally, we should stress that the impurity measurements are a continuing experimental program. As discussed above, we will continue to use time-resolved measurements of the thermal barrier region to check for accumulation of impurities. Wall conditioning experiments during thermal 


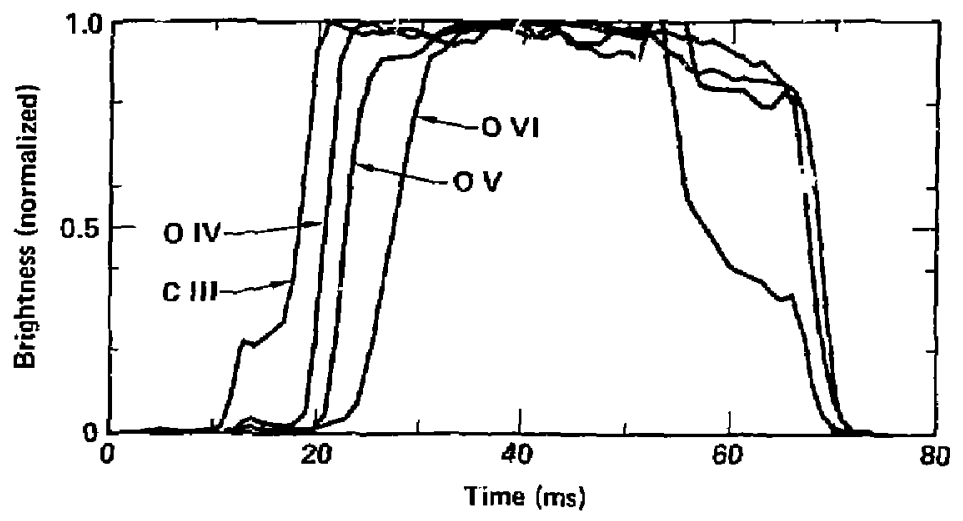

Figure 13, Inpurity emissions versus brightness time (normalized) measured from the end plug of TMX-U during low-power ECRH operation. These data indicate qualitatively that the electron temperature is increasing in the early part of the plasma shot. 
barrier operation are planned; spectroscopic data and residual gas analysis are very important for the interpretation of these results. Recentiy, nitrogen neutral beams were used in the central cell to inject impurities. The amount of injected impurity was small enough so that the plasma was not perturbed. This data is currently undergoing analysis and should provide information on impurity behavior in the central cell of TMX-U.

Additional diagnostic instruments are also planned for $T M X-U$. A radial and spectral imaging spectrograph is planned for arrivai sometime late in 1984, in a cooperative effort with E-Division at LLNL. This instrument should have sufficient wavelength resolution so that radially resolved Doppler profiles can be obtained. These data should provide information on plasma motion such as rotation in the central cell. Further experirists concerning measurement of the neutral deuterium einission, particularly in the region where the plasma is fueled, are also planned. 
APPENDIX A

Calculation of Impurity Densities and Concentrations

from Spectrograph Data

\section{Calculation of the Brightness}

The signal measured by a spectrometer can be used to calculate the physically meaningful quantity called the brightness-B which is proportional to the number of photons per second emitted by the plasma at a given wavelength. Assuming that the plasma is optically thin, the spectrometer receives photons from the entire line of sight of plasma and integrated over the area of plasma subtended by the entrance slit. If we take into account the efficiency of the optics and detector, the brightness may be expressed as

$$
B=\frac{(N / t)}{(Q T) \Omega A}
$$

where $B=$ brightness of the $]$ ine in photons $\mathrm{s}^{-1} \mathrm{~cm}^{-2} \mathrm{sr}^{-1}$,

$\mathbf{N}=$ number of counts integrated over the spectral width of the line in one integration perfod,

$t$ = integration period in seconds,

QT = overall efficiency of the instrument at the position of the line in number of counts/photon ( $Q$ is the quantum efficiency of the detector and $T$ is the grating transmissionl,

$\Omega=$ solid angle of the light entering the instrument determined by the pre-slit and entrance slit used,

$A=$ area of entrance slit.

The instruments are absolutely calibrated so that QT as a function of wavelength is known. We can find $N$ by summing up the signal in the line or by "fitting" the line with a Gaussian function.

\section{Estimation of Impurity Densities}

Each instrument views the plasma along a radial chord. A particular Erilission line from the spectrograph corresponds to a particular ionization state of an impurity species in the plasma. The brightness $B_{s i}$ and the density $n_{s i}$ of the $i$ th ionization state of species " $s$ " are related by 


$$
B_{s i}=\frac{7}{4 \pi} \int n_{s j}(r) n_{e}(r)\langle o v\rangle_{s i} d l,
$$

where $n_{e}(r)$ is the electron density and <ov $\rangle_{s i}$ is the corresponding electron-impact excitation rate. Hote that $\langle 0 v\rangle_{s i}$ is a function of the electron temperature, and hence radius. The values used were obtained frorr LASL report by Merts et a]. ${ }^{30}$ If <ov ${ }_{5}$ i was not listed in the LASL report, values were obtained from a semi-empirical formula $a^{31}$

$$
\langle\sigma v\rangle_{i}=\frac{1.6 \times 10^{-5} f_{i k}\langle g\rangle e^{-E_{i} / T_{e}}}{E_{i}\left(T_{e}\right)^{T / 2}},
$$

where $f_{i k}$ is the oscillator strength for the given transition, $\langle g\rangle$ is the average Gaunt factor (a semi-empirical correction factor), $E_{i}$ is the transition energy, and $T_{e}$ is the electron temperature.

In Eq. (2), the integral needs to be performed along the line of sight of the instrument (say $x$ axis). For convenience of notation, we consider one species and 'rop the index $\underline{s}$ in Eq. (2). The integrand in Eq. (2) is a function of radius. Earn 1024-channel instrument measures the average brightness of one radial chord, and only limited shot-by-shot radial scans were taken. Therefore, we use the model of Refs. 32 and 12 to calculate the impurity density from the brightness. This model is in qualitative agreement with the experimentally obtained radial impurity measurements on TMX. In this model, we make the following assumptions:

(a) The electron density $n_{e}(r)$ is Gaussian with $1 / e$ radius $r_{p}$. This is often a good approximation to the central-cell density profile

$$
\left(i . e ., n_{e}(r)=n_{o e} e^{-r^{2} / r_{p}^{2}}\right) \text {. }
$$

(b) The ion-density radial profile is given by

$$
r_{i}(r)= \begin{cases}n_{i}=\text { constant } & \text { for } r \leq r_{p} \\ 0 & \text { for } r>r_{p}\end{cases}
$$


With this constant impurity density mode?, Eq. (1) becomes:

$$
B_{i} \imath \frac{n_{j}}{4 \pi} \int_{-r_{p}}^{r_{p}} n_{e}(x)\langle 0 v\rangle_{j} d x .
$$

In most cases considered here, <ov $\rangle_{i}$ is a smooth function of electron temperature $T_{e}$. It is nearly constant for $T_{e}$ in the range 30 to $150 \mathrm{eV}$. If there is no sharp change (by a factor of 3) in $T_{e}$ for $r \leq r_{p}$, then we can further approximate $\langle 0 v\rangle_{i}$ by its value at $x=0$ and, since it is a constant, it can be taken out of the integral of (3). With the help of assumption (a), Eq. (3) can then be written as

$$
B_{i}=\frac{n_{i}}{4 \pi}\langle\sigma V\rangle_{i} \times 0.84 \int n_{e} d \ell,
$$

where $s n_{e} d l$ is measured by beam attenuation detectors (BAD) or by a microwave interferometer, and we have used

$$
\int_{-1}^{1} e^{-x^{2}} d x=0.84 \int_{-\infty}^{\infty} e^{-x^{2}} d x
$$

Rearranging (4) we have

$$
n_{i} \simeq \frac{15 B_{i}}{\langle\sigma v\rangle_{j} \int n^{d 2}}
$$

The total number of particles corresponding to this ionization state is

$$
\begin{aligned}
N_{i} & =L \int_{0}^{\infty} n_{i}(r) 2 \pi r d r, \\
& =L \int_{0}^{r} p n_{i} 2 \pi r d r, \\
& \simeq L \pi r_{p}^{2} n_{i},
\end{aligned}
$$

While the total number of deuterium ions (this equals the total number of el. strons in the limit of a trace irapurity) is 


$$
\begin{aligned}
N_{D} & =L \int_{0}^{\infty} n_{e}(r) 2 \pi r d r, \\
& \simeq L \int_{0}^{\infty} n_{o e} e^{-r^{2} / r^{2}} 2 \pi r d r, \\
& =L \pi r_{p}^{2} n_{\text {oe }},
\end{aligned}
$$

where $L$ equals length of the plasma in central cell.

\section{Calculation of Impurity Concentration}

As 1ong as the impurity density is smal1, the plasma (deuterium) ion density is equal to the electron density, and the "global avarage" concentration of this ian state is defined to be

$$
\begin{aligned}
C_{i} & =\frac{\text { total no. of particles in a particular ionization state }}{\text { total no. of deuterium ions }}, \\
& =\frac{N_{i}}{N_{D}}, \\
& =\frac{n_{i}}{n_{0 e}} .
\end{aligned}
$$

With

$$
n_{o e}=\frac{\int n_{e} d l}{\sqrt{\pi} r_{p}}
$$

and $\mathrm{Eq}$. (5), we can express Eq. (8) as

$$
c_{i}=\frac{n_{i}}{n_{p e}}=\frac{15 \sqrt{\pi} r_{p}}{\left[\int n_{e} d \ell\right]^{2}} \frac{B_{i}}{\langle o v\rangle_{i}} .
$$


If a species $\underline{s}$ has seviza? ionization states $\underline{i}$ (including metastable states), then the total concentration $C_{s}$ is the sum of the concentration of each of the states $C_{j}$. Note that the total density of a particular ionization state is composed of both the ground-state population and the metastable-state population. This is because the radiative decay from the metastable state (triplet) to the ground state (singlet) is forbidden from quantum mechanical selection rules. In many cases, the density in each system is nearly equal, i.e.,

$$
c_{s}=\sum_{i} c_{s i}=\frac{\sum_{i} n_{s i}}{n_{\text {oe }}} .
$$

Power Loss Resulting from Radiation

The power loss $P_{j}$ resulting from the jth emission line of a particular species with wavelength $\lambda_{j}$ is given by

$$
p_{j}=\frac{i k c}{\lambda_{j}} \int_{\substack{\text { plasma } \\ \text { volume }}} \text { (volume emission rate) dv, }
$$

where volume emission rate is the number of photons emitted by the plasma per unit time per unit volume at a particular wavelength; or by

$$
\begin{aligned}
& \left.P_{j}=\frac{h c}{\lambda_{j}} \int_{\substack{\text { plasma } \\
\text { valume }}} n_{j}(r) n_{e}(r) \operatorname{cov}\right\rangle_{j} d v, \\
& \left.=\frac{h c}{\lambda_{j}}(2 \pi L) \int_{0}^{\infty} n_{j}(r) n_{e}(r)<\sigma v\right\rangle_{j} r d r, \\
& \left.\approx \frac{h c}{\lambda_{j}}(2 \pi L) n_{j}<\sigma v\right\rangle_{j} \int_{0}^{r} p_{e} n_{e}(r) r d r, \\
& \approx 16.9 \frac{h c}{\lambda_{j}} L r_{p} B_{j}
\end{aligned}
$$


In obtaining Eq. (11), we used assumptions (a) and (b) discussed earlier. The total power ioss is the sum of all radiation lines of all species, i.e.,.

$$
\text { Total } P=\sum_{S} \sum_{i} P_{s i} \text {. }
$$

Exper imental Data of Shot 12 on August 12,1982

By using the method discussed above, we can estimate the concentration of the impurities and power loss on a typical shot, such as Shot 12 on August 12 , 1982.

$$
\begin{aligned}
& \text { At } \mathrm{t}=40 \mathrm{~ms} \text {, the parameters are } \\
& T_{\mathrm{e}}=91 \mathrm{eV} \pm 30 \mathrm{eV} \text { (at } \mathrm{t}=45 \mathrm{~ms} \text {, from Thomson scattering), } \\
& R_{p} \simeq 18 \mathrm{~cm}(\text { at } t=50 \mathrm{~ms}, \text { from } B A D), 33 \\
& \int n e^{d l}=1.35 \times 10^{14} \mathrm{~cm}^{-2} \text { (at } t=50 \mathrm{~ms}, \text { f-oli BAD), } 33 \\
& \mathrm{~L} \simeq 500 \mathrm{~cm}, \\
& n_{a e}=\frac{\int n_{e} d x}{\sqrt{\pi} r_{\beta}}=4.23 \times 10^{12} \mathrm{~cm}^{-3} \text {. }
\end{aligned}
$$

The results of the above calculations are presented in Table A.1. A comparison with TMX is made in Table A.2.

In the above calculations, we include all the ground states as well as - le metastatle states observed. However, we have not included the ionization states $C(V), N(V I)$, and $O(V I I)$. The wavelengths of the emissions from these states are outside the wavelength range of the NIS and were not observed by the GIS.

\section{Discussion of Results}

In Table A.2, the results from TMX are taken from the Ref. 31 . The uncertainties in the concentration and power lass are by about a factor of 2 . 
Table A.l. Experimental data for Shot 12. The quantities are evaluated at $t=40 \mathrm{~ms}$ during the shot.

\begin{tabular}{|c|c|c|c|c|c|c|}
\hline $\begin{array}{l}\text { Ioni- } \\
\text { zation } \\
\text { state }\end{array}$ & $\begin{array}{l}\text { Wave- } \\
\text { length } \\
(\AA)\end{array}$ & $\begin{array}{c}\text { Brightness } \\
\left(10^{15} \text { ph } \mathrm{cm}^{-2} \mathrm{~s}^{-7} \mathrm{sr}^{-1}\right)\end{array}$ & $\left(10^{-8} \mathrm{~cm}^{3} \mathrm{~s}^{-1}\right)$ & $\left(10^{9} \mathrm{cll}^{-3}\right)$ & $\begin{array}{l}\mathrm{n}_{i} / \mathrm{n}_{\mathrm{Ce}} \\
(100 \%)\end{array}$ & $\begin{array}{c}P_{i} \\
\left(10^{2} W\right)\end{array}$ \\
\hline$C(I V)$ & $1548 / 7550$ & 1.27 & 5.89 & 2.40 & $0.06 \%$ & 2.47 \\
\hline$N(V)$ & 1241 & 1.99 & 3.70 & 5.79 & $0.14 \%$ & 4.78 \\
\hline$N(I V)$ & 923 & 0.08 & 1.59 & 0.56 & $0.01 \%$ & 0.26 \\
\hline$N(I V)$ & $765^{a}$ & 0.37 & 4.67 & 0.89 & $0.02 \%$ & 1.46 \\
\hline \multicolumn{2}{|c|}{ Total N } & & & & $0.17 \%$ & 6.5 \\
\hline$H(I)$ & 1216 & 0.22 & 3.21 & 0.76 & $0.02 \%$ & 0.54 \\
\hline $0(V I)$ & $1032 / 1038$ & 4.90 & 2.62 & 20.78 & $0.49 \%$ & 14.26 \\
\hline$O\langle V\rangle$ & $760^{\mathrm{a}}$ & 0.70 & 1.6 & 4.85 & $0.11 \%$ & 2.17 \\
\hline$O(I V)$ & $625^{b}$ & 0.04 & 0.6 & 0.83 & $0.02 \%$ & 0.19 \\
\hline$O(V)$ & $630^{b}$ & 1.79 & 2.91 & 6.83 & $0.75 \%$ & 8.46 \\
\hline$D(I V)$ & 555 & 0.22 & 1.47 & 1.66 & $0.04 \%$ & 7.79 \\
\hline$O($ IV $)$ & 790 & 0.15 & 1.12 & 1.49 & $0.04 \%$ & 0.57 \\
\hline \multicolumn{2}{|c|}{ Total 0} & & & & $0.82 \%$ & 27.54 \\
\hline
\end{tabular}

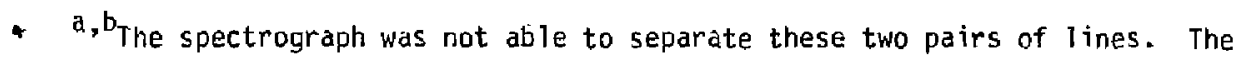
contribution of each 1 ine was calculated. 
Table A.2. Comparison of impurity concentrations and power loss for central cell of TMX and of TMX-U.

\begin{tabular}{|c|c|c|c|c|}
\hline \multirow[t]{2}{*}{ Species $^{\mathrm{a}}$} & \multicolumn{2}{|c|}{ Impurity conrontration (\%) } & \multicolumn{2}{|c|}{ Power loss $(\mathrm{kWl})$} \\
\hline & TMX & $T M X-U$ & $T M X$ & $T M X-U$ \\
\hline Carbon & $0.08 \%$ & $0.06 \%$ & 1.5 & 0.3 \\
\hline Nitrogen & $0.10 \%$ & $0.20 \%$ & 3.5 & 0.7 \\
\hline 0xygen & $0.40 \%$ & $0.80 \%$ & 6 & 2.7 \\
\hline Titanium & $0.10 \%$ & None & 10 & None \\
\hline Deuterium & & & 6 & 0.1 \\
\hline
\end{tabular}

The He-like $C V, N V I$, and 0 VII are not included in the above table.

Nevertheless, from Table A.2 we see that the impurity concentrations in TMX-U are low and are similar to those in TMX. The power loss from EUV radiation is low when compared to the input power and when compared to TMX. The concentration of oxygen is higher in $T M X-U$ while the power loss resulting irom oxygen is lower in TMX-U. This may be due to the electron temperature on these two shots. The temperature in TMX-U Shot 12 is about $90 \mathrm{eV}$ while the " temperature in TMX for data shown in Table A.2 is about $50 \mathrm{eV}$. An iscrease in temperature in this range will lead to a decline in radiated power.

Note that here we have considered lines observed in the region 200 to $1800 \mathrm{~A}$. The emissions of C V, N VI, and O VII are in the range from 20 to $200 \mathrm{~A}$. So far, the emissions fron these conization states have not been observed by the GIS. Nevertheless, these lines are very weakly excited at the current electron teinperature, so there may be some density in these ionization states but very little radiation.

If we make a rough estimate of $C V, N$ VI, and 0 VII from the abundance of the other ionization states, we obtain $0.9 \%$ carbon, $0.5 \%$ nitrogen, $0.9 \%$ oxygen. The uncertainty is about a factor of 3 for carbon and nitrogen and a 
factor of 2 for oxygen. Note that the power radiated by the He-like $C(V)$, $N V I$, and 0 VI will not contribute more than $20 \%$ to the total power loss. This again is because the tiransitions are very difficult to excite at the current electron temperature. 
T. E. Hinnov, Princeton Plasma Physics Laboratory, Report MATT-194, Princeton, NJ (1962).

2. K. Bol et al., in Proc. 5th Int. Conf. Plasma Phys. and Controlled Fusion Res., Tokyo, 1975 (1975), Voi. 1, p. 83.

3. TFR Group, "Space- and Time-Resolved Study of Impurities in TFR Tokamak Plasmas", Phys. Rev. Lett. 36, 1306 (1976).

4. D. Grove et a1., in Proc. 6th Int. Conf. P1asma Phys. and Controlled Fusion Res., Bertchesgaden, 1977 (1977) Vol. T, p. 26.

5. J.L. Terry et al., Nuc?. Fusion 18, 485 (1978).

6. F.H. Coensgen et ai., TMX-U Major Project Proposal, Lawrence [ivermore National Laboratory, LLNL-Prop.-172.

7. F.H. Coensgen et ai., Phys. Rev. Lett. 35, 1501 (1975).

8. F.H. Coensgen et a1., TMX Major Praject Proposal, Lawrence Livermore National Laboratory, L.LNL-Prop.-148.

9. F.H. Coensgen et al., Phys. Rev. Lett. 44, 1132 (1980).

10. R.P. Drake and W.H. Moos, Nucl. Fusion 19, 407 (1979).

11. R.P. Drake and H.W. Moos, Nucl. Fusion 20, 599 (1980).

12. R.P. Drake, Extreme U7traviolet Diagnos is of a Neutral-Beam-Heated Mirror Machine, Lawrence Livernore National Laboratory, UCRL -52751 (1980).

13. S.L. AlTen, 0.T. Strand, H.H. Moos, R.I. Fortner, T.J. Nash, and D.D. Dietrich, Lawrence Livermore Nationa] Laboratory, UCID-18883 (1981).

14. O.T. Strand, H.W. Moos, and S.L. Allen, Nuc7. Fusion 22, 657 (1982).

15. 0.T. Strand, H.W. Moos, and S.L. AT7en, Muct. Fusion 23, 1675 (1983).

16. D.E. Baldwin and B.G. Logan, Phys. Rev. Lett. 43, 1318 (1979).

i7. J.E. Osher, D.P. Grubb, and P. Poulsen, BuTT. Am. Phys. Soc. 28, 1119 , paper $4 \mathrm{R} 12$ (1983).

18. R.K. Richards, H.W. Moos, and S.L. Allen, Rev. Sci. Instrum. 51, 1 (1980).

19. S.L. Allen, Ph. D. Thesis, The Johns Hopk ins University, Baltimore, MO (1980).

20. R.E. Be11, M. Finkentha11, and H.W. Moos, Rev. Sci. Instrumt. 52, 1806 (1981). 
21. T.J. Nash, S.L. Allen, D.D. Dietrich, and R. Fortner, Bull. Am. Phys. Soc. 27, 997, paper 40-8 (1982).

22. T.C. Simonen, S.L. Allen, T.A. Casper et a1., Phys. Rev. Lett. 50, 1668 (1983).

23. T.L. Yu, S.L. Allen, H.W. Moos, and R. Zimmerman, Bull. Am. Phys. Soc. 27, 999, paper 40-18 (1982).

24. S.L. Alten and R.H. Munger, Bul1. Am. Phys. Soc. 27, 991, paper 40-13 (1982).

25. R. Kane and P. Poulsen, privat.e communications, 1983.

26. S.L. Allen, C.A. Clower, R.P. Drake et al., J. Vac. Sci. Tech. A I, 916 (1983).

27. S.L. Allen, C.A. Clower, and W.C. Turner, Bul1. Am. Phys. Soc. 28, 1114 paper 4Q-1 (1983).

28. T.L. Yu, S.L. ATlen, and H.W. Moos: Bu11. Am. Phys. SOC. 28, 1118, paper 4R4 (1983).

29. D.P. Grubb, BuT7. Am. Phys. Soc. 28, 1080 paper 3A-1 (1983).

30. A.L. Merts et a]., Los Alamos National Laboratory, LA-8267-MS (1980).

31. A. Gabriel and C. Jordan, in Case Studies in Atamic Collision Physics,

E. McDaniel and M. Mcoowel1, Eds. (North-Holland, Amsterdam, 1971), Vol. 2, p. 209.

32. O.T. Strand, A Study of Impurities in the TMX Using EUV Spectroscopy, Lawrence Livermore National Laboratory, UCRL-53295 (May 1982).

33. J. Foote, private communications, 1982.

$4295 v / D B / M M$ 